\title{
Epilepsy surgery, visual fields, and driving: a study of the visual field criteria for driving in patients after temporal lobe epilepsy surgery with a comparison of Goldmann and Esterman perimetry
}

\author{
H Manji, G T Plant
}

\begin{abstract}
Twenty four patients who had undergone temporal lobe surgery for epilepsy were assessed to determine $(a)$ whether or not they had developed a visual field defect and $(b)$ if a field defect was present, were the visual field criteria, as required by the DVLA, fulfilled using the monocular Goldmann perimeter test and the automated binocular Esterman method performed on a Humphrey perimeter. A field deficit was found in 13 of 24 (54\%) using the Goldmann perimeter and 11 of 24 $(46 \%)$ by the Esterman method. The second was a more lenient assessment with six of $24(25 \%)$ failing the driving criteria compared with 10 of $24(42 \%)$ by the monocular Goldmann method. Three patients were seizure free but failed the driving criteria. This complication of surgery for temporal lobe epilepsy needs to be discussed with patients before surgery. (F Neurol Neurosurg Psychiatry 2000;68:80-82)
\end{abstract}

Keywords: epilepsy surgery; visual fields; driving

Surgical treatment for patients with temporal lobe epilepsy that is resistant to medical therapy is now an important therapeutic option. For younger patients in particular, the issue of driving is an important one in terms of social independence and in the workplace and may play an important part in the decision to undergo surgery. The complications of surgery for temporal lobe epilepsy include memory and visual field deficits, dysphasia, and contralateral hemiparesis. The development of such complications is dependent on both the nature of the underlying lesion but also the type and extent of surgical resection undertaken.

In the United Kingdom, the minimal field of vision requirement for holding a group one driving licence as required by the Driving and Vehicle Licensing Agency (DVLA) is defined as "at least $120^{\circ}$ width on the horizontal and of at least $20^{\circ}$ from the central fixation point above and below the horizontals on any meridian, measured by perimetry using a $3 \mathrm{~mm}$ white test object at one third metre (or equivalent perimetry)." On the Goldmann perimeter, this equates to the target III ( $4 \mathrm{~mm})$ and filters $4 \mathrm{e}$ setting. In addition, the regulations state "that the binocular field of vision obtained by testing with both eyes open is acceptable although testing individual eyes is useful as this indicates the extent of the field in each eye and is preferable with bitemporal visual field defects."

This study was therefore set up to determine the proportion of patients who have undergone surgery for temporal lobe epilepsy who fail the visual field criteria for driving defined above. This would make them ineligible to drive even if they were rendered seizure free.

\section{Methods}

The records of all patients under the care of the epilepsy service at The National Hospital for Neurology and Neurosurgery who had undergone temporal lobe surgery between June 1986 and June 1995 were obtained. Those who lived within 25 miles of the hospital were contacted. The issue of the DVLA driving regulations and visual field deficits was not raised in the invitation letter. Three patients were referred directly to the ophthalmology department for a visual field assessment as the issue of driving had been raised. Forty patients met all criteria and 21 agreed to take part in the study.

All patients were seen by us and asked about symptoms which would suggest the presence of a visual field defect. The visual fields were assessed by confrontation using finger movements. Subsequently, monocular fields were plotted by the Goldmann kinetic perimeter at the III $4 \mathrm{e}$ setting by one of us. This was

Results of all patients and those with hippocampal sclerosis (HS) only

\begin{tabular}{lcl}
\hline & $\begin{array}{c}\text { All patients } \\
(n=24)\end{array}$ & $\begin{array}{l}\text { HS only } \\
(n=20)\end{array}$ \\
\hline Presence of field defect: & & \\
$\quad$ Clinical & $5 / 24(21 \%)$ & $5 / 20(20 \%)$ \\
$\quad$ Goldmann & $13 / 24(54 \%)$ & $11 / 20(55 \%)$ \\
$\quad$ Esterman & $11 / 24(46 \%)$ & $10 / 20(50 \%)$ \\
$\begin{array}{l}\text { Field defect and failed driving criteria: } \\
\quad \text { Goldmann }\end{array}$ & $10 / 24(42 \%)$ & $8 / 20(40 \%)$ \\
$\quad \begin{array}{l}\text { Esterman } \\
\text { No seizures, but failed } \\
\text { driving criteria }\end{array}$ & $7 / 24(29 \%)$ & $6 / 20(30 \%)$ \\
$\quad$ (Esterman): & $3(12.5 \%)$ & $3(15 \%)$ \\
\hline
\end{tabular}


A
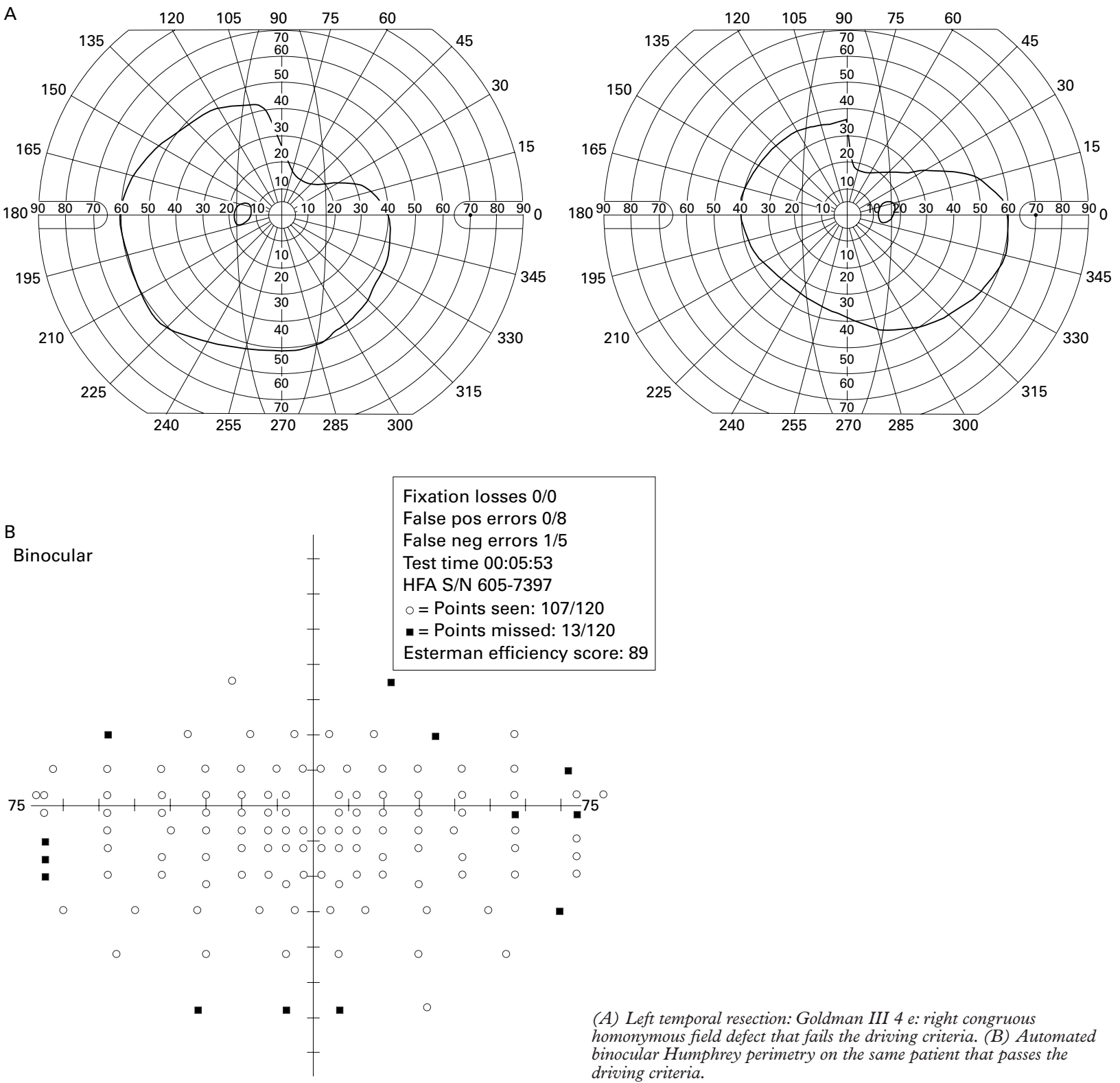

followed by an automated Esterman binocular field assessment charted on a Humphrey perimeter. When appropriate, a visual acuity correction was used. The patients' hospital records were later reviewed to determine their seizure control as recorded by their physicians in the follow up epilepsy clinics.

\section{Results}

In all, 24 patients were studied (table): 13 women and 11 men with a mean age of 33.3 years. The pathological diagnoses were hippocampal sclerosis (20 patients), non-specific gliosis (one), dysembryoplastic neuroepithelial tumour (one), gliosis after cerebral abscess (1). The surgery was performed by two surgeons (WH, who performed 19 of 20 of the HS operations and one of four of the nonhippocampal sclerosis operations and DT, who performed 1 of 20 of the hippocampal operations and three of four of the nonhippocampal sclerosis operations.
A review of the surgical preoperative notes showed that no patient had symptoms suggestive of a field defect or had a documented field defect as determined by confrontation methods. The visual acuity was recorded in 21 patients - this was $6 / 6$ or better (with or without correction) in all.

Only one patient, who failed the driving criteria, had symptoms suggestive of a field defect before the field analyses. The field defects were only detected clinically in five out of the 24 patients $(21 \%)$. These were all patients whose defects were large enough to fail the driving criteria.

Four patients failed on the monocular Goldmann test but passed on the binocular Esterman perimetry test (figure). There was no difference between the hippocampal sclerosis and non-hippocampal sclerosis groups and the presence of a field defect or the numbers who failed the driving criteria. No significant correlation was found between those passing or 
failing the driving criteria and either the side of operation or those who had become seizure free.

\section{Discussion}

The geniculocalcarine tract fibres radiate from the lateral geniculate body round the temporal horn of the lateral ventricle to the ipsilateral occipital cortex. The most anterior and inferior fibres, the so called Meyer's loop, from the upper visual quadrant are the most vulnerable during temporal lobe surgery and damage results in a homonymous superior quadrantanopia. Infarction in the territory of the anterior choroidal artery resulting in a homonymous hemianopia has also been reported in patients after temporal lobe surgery for epilepsy. ${ }^{3}$

The prevalence of visual field defects after temporal lobe surgery for epilepsy ranges from $52 \%$ to $95 \%$. $^{23}$ However, these studies are not comparable as they all used different methods of visual field assessement including automated static Humphrey perimetry, kinetic Goldmann perimetry using targets of varying sizes and intensities, and in one study, the Bjerrum screen. The Newman study concluded that visual field defects are common, rarely dense (in about $2 \%$ ), and usually not of functional relevance although this second phrase was not defined. ${ }^{4}$ The study by Tecoma et al also suggested that the presence of a visual field defect was associated with a good outcome in terms of seizure control. ${ }^{2}$

The study we report here is the first to consider the issue of visual field deficit with respect to driving after surgery for temporal lobe epilepsy. The results are only applicable to the United Kingdom as the visual field criteria for driving vary between different licensing authorities around the world. In this study, 54\% of patients had a field defect using the Goldmann monocular method of assessment and $46 \%$ using the binocular Esterman method. This compares well with the published data from other centres. However, in $25 \%$ of patients, using the less sensitive binocular Esterman assessment, the defect was large enough to fail the current visual field criteria as recommended by the DVLA. A small proportion of these subjects were now seizure free but unable to drive because of their field defect.

The Esterman binocular field assessment performed on a Humphrey perimeter is clearly more lenient than the monocular Goldmann as in this study four patients passed on the first and failed on the second test. Although bowl luminance is the same in both tests at $31.5 \mathrm{Asb}$ and the target size is equivalent, the target luminance is $1000 \mathrm{Asb}$ in the Goldmann method but 3150 Asb in the Humphrey test. The other major difference is that the Goldmann method is a kinetic whereas the Esterman test is a static assessment.

These results, however, need to be viewed in the context of the development of the field of epilepsy surgery at this hospital; the earliest patients in this study were operated on in 1986 when few operations were being performed. The last patients recruited were operated on in 1995 when 40 to 50 operations were being performed annually. During this period a change in surgical technique favoured smaller neocortical resections. In this series there was a trend towards fewer significant field defects in the patients recruited later on in the study. In 1992, two out of five $(40 \%)$ of patients with hippocampal sclerosis failed the driving criteria compared with two out of eight $(25 \%)$ in 1993.The numbers are too small for statistical analysis. Clearly, to obtain figures that reflect current practice it is necessary to undertake a further prospective study.

The methodology of this present study may be criticised on several grounds. Only half of the eligible patients agreed to being assessed, thus increasing the scope for a self referral bias. The issue of visual field deficits and driving was not intimated in the introductory letter making it unlikely that patients who were driving declined to attend. A more likely explanation for the poor uptake is that this group of patients is already being subjected to close scrutiny after surgery with repeat imaging studies, neuropsychological, and psychiatric assessments, which are time consuming.

The fields were not accurately charted by formal perimetry before surgery raising the possibility that some patients had a significant defect as a consequence of the underlying lesion rather than the surgical procedure. Confrontation methods are relatively insensitive as shown by the fact that even in this study, only five patients were found to have an abnormality by this method.

In summary, therefore, this study suggests that an appreciable proportion of patients undergoing surgery for temporal lobe epilepsy develop visual field defects. The exact percentages vary with the type of assessment utilised. In around $25 \%$ of these patients the defect is large enough to fail the current field of vision criteria recommended by the DVLA. These possibilities need to be discussed as part of the preoperative counselling of patients. Further studies are now necessary to take into account the increased expertise gained by neurosurgeons who are performing these operations more often, with more refined techniques and more selective procedures.

We thank all the patients who agreed to take part in the study and Professor S Shorvon, Professor J Duncan, Dr D Fish, Professor D Thomas, and Mr W Harkness for allowing us to study their patients.

1 For Medical Practitioners: at a glance guide to the current medical standards of fitness to drive. Swansea: DVLA Drivers Medical Group.

2 Tecoma ES, Laxer KD, Barbaro NM, et al. Frequency and characteristics of visual field deficits after surgery for mesial characteristics of visual field deficits after surgery

3 Hughes TS, Lavin PJ, Fakhoury AK, et al. Prospective quantitative visual field analysis in epilepsy patients undergoing temporal lobe resection [abstract]. Neurology 1997; 48:A42.

4 Newman SA. Epilepsy surgery for partial complex seizures. A prospective study of afferent visual system pathology [abstract]. Invest Ophthalmol Vis Sci 1995;36:S681. 